\section{$\underset{\substack{\text { hommes } \\ \text { \& migrations }}}{ }$}

\section{Hommes \& migrations}

Revue française de référence sur les dynamiques

migratoires

$1322 \mid 2018$

Exposer les migrations

\title{
Parcours de migrations.
}

Une collaboration entre l'Écomusée Creusot Montceau et la Compagnie Zumbò

\section{Typhaine Le Foll et Matías Chebel}

\section{(2) OpenEdition}

\section{Journals}

\section{Édition électronique}

URL : https://journals.openedition.org/hommesmigrations/6731

DOI : 10.4000/hommesmigrations.6731

ISSN : 2262-3353

Éditeur

Musée national de l'histoire de l'immigration

Édition imprimée

Date de publication : 1 juillet 2018

Pagination : 147-155

ISBN : 978-2-919040-42-1

ISSN : 1142-852X

\section{Référence électronique}

Typhaine Le Foll et Matías Chebel, «Parcours de migrations. », Hommes \& migrations [En ligne], 1322 | 2018, mis en ligne le 01 juillet 2020, consulté le 21 janvier 2022. URL : http://journals.openedition.org/ hommesmigrations/6731; DOI : https://doi.org/10.4000/hommesmigrations.6731 


\title{
PARCOURS DE MIGRATIONS UNE COLLABORATION ENTRE L'ÉCOMUSÉE CREUSOT MONTCEAU ET LA COMPAGNIE ZUMBÓ
}

Par TYPHAINE LE FOLL, directrice de l'écomusée Creusot Montceau, et MATÍAS CHEBEL, directeur artistique de la Compagnie Zumbó.

\author{
Situé au cœur de l'ancien bassin industriel de Saône-et-Loire, \\ l'Écomusée Creusot Montceau développe des collaborations \\ artistiques et une démarche participative visant à sauvegarder \\ la mémoire vivante d'un territoire nourri par les migrations. \\ Conduit par la Compagnie Zumbó à partir des collections \\ de l'écomusée et de témoignages collectés auprès d'habitants \\ du territoire, le projet Murs/murs a contribué à interroger et à \\ renouveler le projet muséal de l'écomusée. Murs/murs a été mis \\ en écho avec le travail photographique d'Hanicka Andrès, \\ mêlant les générations, suscitant des rencontres et le partage \\ des histoires.
}

Creusot, Montceau, Montchanin, Écuisses, Blanzy, Ciry-le-Noble... autant de communes dont les histoires fondatrices puisent leurs racines dans la révolution industrielle du XIX ${ }^{\text {e }}$ siècle. Autant de lieux géographiques qui, dans une France en pleine expansion économique, ont aimanté les rêves d'Arcadie de populations parfois modestes, voire démunies.

Aujourd'hui encore, ce territoire se peuple de nouveaux arrivants nourris d'un profond désir de vie meilleure. Tous, quels qu'ils soient et quelles que soient leurs motivations, ont vécu des expériences migratoires ponctuées de traumas et de nouveaux bonheurs. De nouveaux défis d'intégration se font jour, qui suscitent des questionnements et des réactions de la société civile, aux niveaux politique, scientifique et artistique. Ces questionnements sont-ils vraiment nouveaux dans un territoire qui s'est, historiquement, construit et enrichi au fil des vagues d'immigrants attirés par les perspectives et les promesses d'embauches des industriels de la métallurgie, de la mine, de la céramique et du textile, pour ne citer que les plus importantes? Ou bien comportent-ils des spécificités qui les distinguent des phénomènes migratoires des siècles précédents?

L'Écomusée Creusot Montceau, créé en 1974 en même temps que la communauté urbaine éponyme, consacre une part importante de son travail scientifique à la sauvegarde de la mémoire des hommes et des femmes qui habitent, qui vivent et qui travaillent dans les trente-quatre communes réunies. Musée emblématique aux yeux de la communauté des professionnels - ethnologues, anthropologues, historiens, conservateurs du patrimoine, etc. - parce que l'un des premiers à avoir été créé par Georges-Henri Rivière, il se définit notamment comme un "...miroir où [la] population se regarde, pour s'y reconnaître, où elle cherche l'explication du territoire auquel elle est attachée, jointe à celle des populations qui l'y ont précédée, dans la discontinuité ou la continuité des générations ${ }^{1}$ ». 
Proposée en 2015, la collaboration avec la Compagnie Zumbó s'est retrouvée projetée dans une actualité migratoire largement relayée par les médias. Cette année, nous assistons à une crise des mouvements migratoires sans précédent. S'il n'est pas possible d'identifier un point de départ, quelques repères permettent de resituer ce contexte : depuis 2011, les indicateurs témoignent d'une hausse progressive de ces mouvements. En 2014, les arrivées sont multipliées par quatre sur les côtes italiennes et grecques. En avril 2015, les événements prennent une tournure vertigineuse. Les facteurs multiplicateurs passent de 1 à 10 : l'Organisation internationale des migrations (OIM) recense plus d'un million d'arrivées en Europe transitant par la mer et la route des Balkans. Une hausse expliquée notamment par les conflits en Afrique, au Moyen-Orient et en Syrie, conjugués à une détérioration des conditions de vie dans les camps de réfugiés syriens en Turquie, au Liban et en Jordanie. Ces migrants sont, pour la plupart, originaires de Syrie, d'Afghanistan, d'Irak, d'Érythrée, du Niger, de Somalie, du Soudan.

À la fois écomusée et musée de société, l'écomusée ne pouvait pas faire l'impasse sur une actualité qui interroge une histoire commune. La population française n'est pas homogène mais profondément cosmopolite, façonnée par des mouvements de population qui ont jalonné son histoire et qui continuent de la modeler au gré d'une géopolitique complexe.

\section{La rencontre d'un projet artistique et d'un projet muséal}

La première rencontre entre l'écomusée et la Compagnie Zumbó a eu lieu en 2015. Arrivés au Creusot une année auparavant, ses fondateurs, Mayleh Sanchez et Matías Chebel, avaient été immédiatement attirés par la richesse de l'histoire locale, et plus particulièrement par les questions migratoires dans la région. Eux-mêmes immigrés, ils avaient commencé à travailler sur une nouvelle forme artistique hybride, mêlant théâtre et vidéo, et cherchaient un espace pour présenter leur projet.

Jusqu'alors, l'écomusée avait toujours fait appel à des ethnologues pour recueillir la parole des habitants. Mais le projet présenté par la Compagnie Zumbó était un projet d'événementiel dont la mise en œuvre devait nous permettre d'interpeller le public pour lui montrer, sans rendre la démonstration pesante, qu'il n'existe pas qu'une seule manière de visiter un musée ou de scénographier des objets de collection accompagnés d'un propos historique ou ethnologique.

Pour Zumbó, il s'agissait de la première collaboration avec un musée et l'idée d'adapter sa proposition artistique aux espaces du Château de la Verrerie (siège de l'écomusée) était très stimulante ${ }^{2}$.

De cette rencontre entre les deux structures est née une collaboration soutenue qui se poursuit encore aujourd'hui. Depuis sa genèse en 2015, contre toute attente et sans préméditation initiale, poussés par l'implication des membres de la compagnie et par les réactions du public, ce projet s'est développé pour devenir une "chambre d'écho » d'histoires de vie.

\section{Traçages: de la poésie dans un lieu de mémoire}

Destiné à donner la parole aux habitants, ce projet répondait à l'une des missions fondamentales de l'écomusée, en lien avec la sauvegarde du patrimoine immatériel du territoire. Cette expérience permettait en outre de proposer au public une 
nouvelle manière de lire sa propre histoire et de renouer avec ses origines et son identité. Mais ce partenariat a pu fonctionner surtout en raison d'une écoute réciproque, d'une confiance et d'un dialogue constant entre l'institution et la compagnie.

Dans cet esprit, l'écomusée a dès le début décidé d'encourager l'expérience en ouvrant ses espaces, ses archives et ses réserves aux artistes de Zumbó, ce qui a enrichi le processus de création et a permis au projet de prendre sa forme définitive, une installation intitulée Traçages.

Depuis sa formation, la compagnie interroge les rapports entre l'individu et la société dans laquelle il évolue. Pour cette nouvelle création, la recherche se concentrait sur quelques questions. Comment, en tant que communautés, transmettre sa propre mémoire? Quelles sont les traces identitaires de la ville? Quelles sont les lectures possibles de son histoire, de sa culture? Quelle est l'histoire en commun des migrants arrivés au Creusot? Quel a été leur point de rencontre? Quel regard portent les Creusotins/Creusotines sur leur ville, sur leur histoire, sur ces migrants ? Serait-il possible de se servir de cette mémoire collective pour estomper les divisions d'aujourd'hui?

La rencontre fortuite avec une vieille dame italienne qui avait fui l'Italie de Mussolini pour construire sa vie au Creusot a été le point de départ de ce travail de collecte. Elle s'appelait MarieAbondance, telle une métaphore de l'apport de la main-d'œuvre étrangère au développement de la région. La Compagnie Zumbó est ainsi partie à la rencontre des habitants et de leurs histoires: quelques-unes anciennes, d'autres plus récentes; chacune différente et passionnante à sa manière. Ces témoignages ont été parfois enregistrés et/ou filmés. Pour cela, la compagnie a invité l'association locale La Baraque $\mathrm{TV}^{3}$ à collaborer. Depuis sa création, cette web TV s'est donné pour mission de mettre en valeur les pratiques artistiques amateurs qui ont trait à la mémoire de leur territoire. Au moment de la rencontre avec Zumbó, ils travaillaient sur un projet autour de la Deuxième Guerre mondiale au Creusot.

Traçages ${ }^{4}$ a été conçu comme le croisement de plusieurs langages artistiques, chacun avec ses codes, pour confronter le public à l'histoire de son territoire, avec plusieurs regards possibles, du plus concret au plus poétique. L'installation se déroulait dans deux ambiances juxtaposées bien distinctes : un espace audiovisuel et un espace théâtral. L'espace audiovisuel présentant un montage des témoignages filmés était le cœur de l'installation. Dans une volonté de dialogue entre mémoire et poésie, des objets provenant des réserves de l'écomusée, normalement inaccessibles au public, ont été placés dans la salle et ont servi de support de projection à des images d'archives.

Dans un musée de France, environ $10 \%$ des collections sont exposées en permanence. $90 \%$ d'entre elles sont conservées en réserve. Pour autant, Traçages a été conçu comme le croisement de plusieurs langages artistiques, chacun avec ses codes, pour confronter le public à I'histoire de son territoire, avec plusieurs regards possibles, du plus concret au plus poétique. elles ne sont pas oubliées et font l'objet de programmes de recherche, d'étude et de conservation ou de restauration. Elles sont aussi soumises, à l'instar de toutes les collections inscrites aux inventaires des musées de France, à des règles de droit qui les protègent de toute action d'échange, de vente ou de saisie. Ces dispositions, qui rendent donc les collections inaliénables, imprescriptibles et insaisissables sont les garants du statut de «patrimoine national » qui leur est conféré dès l'inscription à l'inventaire. Dans sa démarche d'exploration des collections en réserve pour sélectionner les objets les mieux à même de résonner avec l'idée de partage d'un patrimoine commun, le travail de la compagnie s'apparentait à celui qui est mis en œuvre par les conservateurs du patrimoine au moment de la conception des expositions temporaires. Sous ce nouvel éclairage, 
Exposition Murs/murs, inaugurée lors des Journées européennes du patrimoine de 2016. Photo Matias Chebel. (c) Matias Chebel/Compagnie Zumbó.

chaque objet jouissait d'une visibilité nouvelle et prenait toute sa place dans ce tissu de récits. Les témoignages vidéo cohabitaient avec un montage de vidéo-art conçu par le réalisateur Ragnar Chacín, à partir notamment d'extraits de films muets tournés dans les usines à l'initiative de l'entreprise Schneider et $C^{\text {ie }}$ au début du XXe siècle et conservés par l'Écomusée Creusot Montceau.

Plus onirique, l'espace théâtral disposait d'un faible éclairage. Dans l'intimité de ce dispositif, un personnage sans âge, intemporel, imprégné de tout cet univers social (ouvrier, minier et migrant), prenait la parole pour plonger le public dans une expérience à la fois sensible et poétique. Le texte a été inspiré par un conte de Gabriel Garcia Márquez, revisité et adapté aux légendes de la Bourgogne.

La transposition de documents d'archives visuelles et d'objets de collections dans un espace à forte théâtralité est ici une métaphore pour suggérer que le réel est en perpétuelle mutation, en devenir, et qu'il se matérialise différemment selon les époques et les cultures. Faits et objets se succèdent, passent et disparaissent. Le patrimoine conservé, étudié et valorisé dans les musées de France est une réponse au temps qui passe, il exprime un désir de continuité qui se traduit par l'analyse et la sélection d'artefacts et de faits culturels, scientifiques, techniques ou naturels qui seront, par tous les moyens disponibles, préservés de la disparition et de l'oubli. 
Au cours des représentations de Traçages, le musée a accueilli un public très varié : publics d'habitués, nouveaux publics n'ayant jamais visité le musée, élèves des collèges et lycées de la communauté urbaine... Les retours ont été positifs et encourageants. Les associations de migrants directement concernés par le projet se sont mobilisées, de même que les familles de ces derniers. Chacun a pu être touché de manière différente, selon son âge, ses centres d'intérêt et sa sensibilité. La transversalité de la proposition a suscité des échanges entre communautés et générations différentes: des petits-enfants ont ainsi, pour la première fois, entendu de la bouche de leurs aïeux l'histoire et les origines de leurs familles.

Un dossier pédagogique, validé par l'équipe de l'écomusée, a été mis à disposition des enseignants avec plusieurs activités suggérées qui allaient de l'analyse de la forme artistique à un travail de recherche. Parmi celles-ci, figurait notamment la proposition faite aux élèves d'aller interviewer à leur tour leurs aïeux en mettant à profit leurs téléphones portables. Le lycée Léon Blum du Creusot a inscrit cette activité au concours « Viva-Cité » organisé par l'Académie de Dijon et a décroché la mention du jury. Les productions des élèves ont fait l'objet d'une présentation à l'écomusée en mai 2016 durant la Nuit des Musées.

\section{Après Traçages : construire une chambre d'écho}

Après Traçages, l'Écomusée Creusot Montceau souhaitait prolonger le travail mené avec la Compagnie Zumbó, sans avoir l'assurance de pouvoir conjuguer les ressources humaines au calendrier et au budget pour transformer les envies en projets.

La Compagnie Zumbó s'interrogeait sur la manière de restituer au public la totalité des témoignages collectés qui, pour des questions de cohérence dramaturgique ou de temps, n'avaient pu trouver leur place dans l'installation Traçages. Matías Chebel, directeur de Zumbó, a proposé le projet Murs/murs : il s'agissait d'organiser des projections géantes sur les façades de l'écomusée, lors de la Nuit des
Musées. Les murs du Château de la Verrerie auraient laissé place aux récits de vies de toutes ces personnes.

Pour l'écomusée, deux priorités sont apparues clairement pour inscrire le projet Murs/murs dans une démarche structurante. Tout d'abord, il devait permettre la poursuite des pratiques de sauvegarde du patrimoine immatériel engagées par l'institution de 1976 à 1978 à travers une série d'enquêtes ethnographiques rassemblées sous le titre Histoires de vies. Pour ce faire, il fallait mobiliser les ressources du territoire et, notamment, une association spécialisée dans la collecte de témoignages oraux et vidéos réalisés sous la forme d'entretiens non directifs conduits par Matías Chebel avec la collaboration de
Au cours des représentations de Traçages, le musée a accueilli un public très varié : publics d'habitués, nouveaux publics n'ayant jamais visité le musée, élèves des collèges et lycées de la communauté urbaine.. nécessaire de retravailler la muséographie de l'une des salles du parcours d'exposition permanente intitulée « Salle des portraits » qui sétait retrouvée dépouillée, au fil des ans, au point de ne plus donner à voir que deux portraits au début de l’année 2012. L'articulation de ces deux projets devait permettre de redonner tout son sens à cette salle, tout en la modernisant.

L'objectif était donc multiple : renouer avec une pratique scientifique de l'écomusée en suivant l'élan donné par la collaboration avec la Compagnie Zumbó ; inscrire la démarche dans un programme de sauvegarde patrimoniale ; tisser un lien entre la structure, un territoire, son histoire et sa population ; introduire des éléments de modernité dans l'approche muséographique en ouvrant la porte à d'autres pratiques culturelles; favoriser la création contemporaine ; faire entrer au musée les sujets de société qui animent le monde contemporain dans lequel évoluent quotidiennement les publics de nos musées.

La problématique des migrations humaines s'est imposée avec évidence pour faire l'objet d'un travail mobilisant résidents, associations locales, acteurs, artistes, médiateurs, techniciens, conservateurs, élus, chercheurs, pour donner forme à un projet 
pluridisciplinaire et protéiforme qui prendrait valeur de chambre d'écho patrimoniale du territoire.

Dans ce sens et dans l'optique de pérenniser l'idée du projet Murs/murs au-delà d'une seule projection nocturne sur les murs du Château de la Verrerie, l'écomusée a invité Zumbó à adapter sa proposition et à investir la « salle des portraits » avec un format d'exposition.

Conçu comme un triptyque réparti sur trois saisons, avec un nouveau chapitre dévoilé

chaque année, Murs/murs cherche à ouvrir une réflexion par rapport à la constitution de nos sociétés et à la manière

dont elles évoluent face aux enjeux contemporains.
Comédien d'origine argentine, Matías Chebel est arrivé en France à la fin de l'année 2001 alors qu'une crise économique sans précédent ravageait son pays. Son histoire personnelle l'a conduit à s'intéresser à celles des femmes et des hommes qui ont contribué à la fabrication de la renommée du territoire Creusot

Montceau parce que, en tant que citoyen, en tant qu'être humain, ces récits racontent aussi sa propre histoire ${ }^{5}$.

Conçu comme un triptyque réparti sur trois saisons ${ }^{6}$, avec un nouveau chapitre dévoilé chaque année, Murs/murs cherche à ouvrir une réflexion par rapport à la constitution de nos sociétés et à la manière dont elles évoluent face aux enjeux contemporains. En traçant un parallèle entre les flux migratoires des différentes époques, ce travail de collecte vidéo de témoignages de migrants met en mémoire et tente d'éclairer la perception sociale, culturelle, politique du sentiment d'appartenance qui prévaut et participe de la mise en œuvre d'une société plus inclusive.

La salle du musée qui était auparavant dédiée aux portraits peints retrouve en quelque sorte sa fonction originelle, à la différence près que, cette fois-ci, les visages s'animent. Ils usent de la parole et invitent à découvrir ces « histoires avant l'histoire », celles de l'arrachement à une terre natale, du changement de condition sociale et des conséquences du déracinement associées à la perte des repères et à la dure réalité de l'usage d'une langue qui, tout à coup, dessert la communication au lieu de la nourrir. Ces récits expriment avec acuité le déroulement d'une vie au jour le jour, dans la nécessité d'être, sans savoir s'il sera possible de se projeter dans un avenir.

La triste actualité et l'emballement médiatique autour de la problématique des migrants depuis l'été 2015 nous invitent à penser qu'aujourd'hui, plus que jamais, ce projet a toute sa place au sein de l'Écomusée Creusot Montceau.

Encouragé par cette collaboration fructueuse avec la Compagnie Zumbó, l'écomusée a choisi de faire appel à d'autres artistes ou structures engagés pour donner davantage de visibilité à la thématique migratoire au sein de ses espaces d'expositions.

L'écomusée a ainsi produit l'exposition Corps de migrants, qui rassemble le travail de l'Atelier du Coin et d'Hanicka Andrès, artiste photographe. Ouverte depuis le 20 mai 2017, l'exposition sera présentée au public jusqu'au 30 novembre 2018.

\section{L'Atelier du Coin : une approche sociale de la migration?}

L'Atelier du Coin est un atelier d'insertion de l'association Arc-en-ciel. C'est une structure qui offre un dispositif de portage de projet dont la vocation est d'aider les bénéficiaires à s'insérer dans un tissu social à travers un projet professionnel.

Il est aussi un lieu de convivialité qui accueille des groupes d'âges et de milieux différents pour leur faire découvrir les techniques de la gravure sur bois, de l'imprimerie à l'ancienne, de la typographie, de la menuiserie et de la céramique.

Suite à la proposition de lécomusée, les membres de l'atelier ont lu et écouté les témoignages présents dans Murs/murs : chaque personne y raconte son 
Exposition Corps de migrants, inaugurée lors des Journées européennes du patrimoine de 2017 à l'écomusée Creusot Montceau. Photo Daniel Busseuil. @ DANIEL BusseuIL.

parcours, parfois difficile, pour accéder à un travail et à un refuge en France. Ensuite, les vingt salariés de l'atelier se sont concertés et ont choisi la symbolique des mains, gravées sur le bois, pour cataloguer les gestes pratiqués par les populations en route vers leur nouvelle destination ou par les autochtones qui les accueillent. Elles disent les épreuves, les dangers rencontrés, "les douleurs", les sentiments des uns, les ressentiments des autres, «la solidarité », « la mixité », «l'espoir [...] d'un travail et d'un refuge ».

Les gravures visibles dans l'exposition évoquent ainsi les sentiments des migrants. Le cour arraché entre les mains raconte comment le fait de partir de son pays peut briser le cœur ; le village à l'arrièreplan est le vrai village italien qui a dû être quitté et qui est aujourd'hui en ruine. Le mélange des mains est une gravure qui raconte la solidarité et la mixité qui ressort aussi des histoires de migrants : chacun arrive de son pays et peut faire partager aux autres sa culture et ses spécialités culinaires. La carte du monde : l'image représente le trajet et l'arrivée en France avec notamment la traversée des pays, de la mer, les différentes étapes parfois longues et difficiles. La valise et les mots qui s'envolent représente les espoirs que chacun peut avoir en quittant son pays et en rejoignant la France : la sérénité, la liberté, l'avenir, le bonheur?. 


\section{Hanicka Andrès : de la déclaration militante de I'artiste à sa production artistique}

Voici ci-dessous le texte rédigé par Hanicka Andrès, lu, enregistré et diffusé dans l'exposition Corps de migrants en regard des douze calicots présentant, in extenso, les douze témoignages de migrants ayant répondu à l'appel ainsi que leurs portraits réalisés par l'artiste.

"2016, le monde poursuit sa marche sur la tête: élection de Donald Trump, l'affaire du Burkini, évacuation de la jungle de Calais, attentat en Turquie, Côte d'Ivoire, Belgique, Allemagne, Tunisie, à Nice, Ouagadougou, Bagdad, au Pakistan ... Pendant ce temps, au Creusot, un ami se voit refuser un logement, au nom de la Laïcité, parce qu'il est musulman...

\section{URGENCE!}

Leur histoire, leur parcours de vie, les a conduits ici, par nécessité, souvent dans l'urgence...

Ils viennent d'arriver en France, ou sont nés en France avec des origines étrangères. Ils sont chrétiens, musulmans, athées, ..., préfèrent le couscous aux spaghettis, les pizzas au cassoulet, ou sont gourmands de tout. Ils parlent parfois plusieurs langues, font tourner une petite entreprise ou travaillent pour un patron, simpliquent dans des associations, éduquent leurs enfants au respect de chacun. Ils ont le projet de partir en vacances, d'acheter une maison, de trouver les ressources nécessaires pour faire réparer la voiture, que le petit dernier puisse faire de grandes études. Ce sont nos voisins, nos collègues, nos amis; ils sont issus de l'immigration italienne, algérienne, portugaise, québécoise, cambodgienne, soudanaise, ..., des citoyens Français ou qui rêvent de le devenir.

Les rencontrer, les écouter, les accueillir, les respecter, les remercier, ... ; ma nécessité, mon urgence!

Cette exposition je la dédie à tous ceux qui ont quitté leur pays d'origine par nécessité, souvent dans l'urgence ;

à mes grands-parents d'origines slovaque et espagnole; à mes merveilleux enfants, Zinédine et Rayan, dont je suis l'heureuse maman. "

Les protagonistes photographiés par Hanicka Andrès sont tous volontaires pour poser devant son appareil : un Leïca équipé d'un optique $35 \mathrm{~mm}$. Les tirages en noir et blanc, les cadrages resserrés, les vues frontales ont quelques similitudes avec les techniques d'investigation de la police des frontières. La mise à distance, au sens propre comme au sens figuré, que la photographe s'impose, la valise, qui abrite les viatiques nécessaires à ces voyages, disent la similitude des situations traversées par tous. Les ambiances de prise de vue complètent les textes et distinguent différents lieux clés dans ces parcours de migration : ils représentent les points cardinaux entre un passé et un présent, entre deux générations.

La méthode artistique développée par Hanicka Andrès dans ce projet, emprunte le hasard pour mettre en lumière une certaine forme de volontarisme. Elle n'est pas sans évoquer les aléas de la vie - on ne choisit pas de naître dans un pays plutôt que dans un autre - et les choix exprimés par les personnes photographiées qui témoignent de leur décision de quitter leur pays, de se couper de leurs racines pour se choisir une autre vie.

Tout comme dans la "vraie vie ", ces femmes et ces hommes ont répondu à la promesse d'un avenir meilleur, ils répondent dans ce projet artistique à l'appel d'une artiste sur les réseaux sociaux. Leur volonté impérieuse de rester acteurs de leurs vies les conduit à émigrer. Dans ce projet, initié via le support immatériel d'un réseau social sur Internet, le choix de se signaler à l'artiste relève d'une volonté de témoigner. Tantôt acteurs, tantôt témoins, tous expriment, à travers ce projet, la nécessaire progression pour prendre du champ par rapport à leur vécu et parvenir, à travers la verbalisation et la mise en récit, à transformer leur histoire et leur témoignage en expérience. Ici, l'artiste met à profit la dématérialisation d'un réseau informatique pour recréer un réseau social. Les protagonistes, d'abord dispersés de par le monde, le long des routes de migrations, installés qui à Torcy, qui à Montchanin, qui au Creusot ou dans les environs, sur un territoire où ils ne se connaissent pas, tissent un lien virtuel avec une artiste sur la base d'un projet lancé sur la toile, à l'image des bouteilles à la mer: ils se signalent, initient ensuite une communication par messagerie avec l'artiste qui leur explique son protocole de création ; ils acceptent les règles de mise en œuvre du projet artistique, rédigent et lui transmettent leurs récits personnels ou familiaux avant de rencontrer la photographe qui va procéder à la mise en portrait. 
Pour un musée d'ethnologie, le projet artistique et les modalités de prise de vue conçus par Hanicka Andrès créent une continuité historique avec les productions des photographes portraitistes du XIX siècle. La toile de fond systématiquement utilisée par ceux qui travaillaient en studio est remplacée par un paysage naturel. Qu'il soit individuel ou collectif, le portrait imagé a pour fonction de résumer le contenu du récit et l'aventure individuelle ou générationnelle narrée par le témoin. Les outils, objets ou instruments du XIX siècle ou du début du XX $\mathrm{XX}^{\text {e }}$ siècle choisis par leurs propriétaires comme attributs symbolisant leurs métiers, leurs passions ou leurs fonctions, sont ici systématiquement remplacés par un bagage qui exprime une condition temporaire, passée ou actuelle, et qui traduit la notion de mobilité propre à cette condition humaine, ainsi qu'une forme de précarité inhérente à l'urgence de la situation et à l'errance. Les estampes produites par les membres de l'Atelier du Coin sont, quant à elles, autant de métaphores qui illustrent et résument les parcours de migration des témoins sélectionnés par l'artiste photographe.

\section{Conclusion}

Ces trois productions rassemblées dans deux expositions juxtaposées et respectivement intitulées Murs/murs et Corps de migrants sont mises en scène de telle manière que le public doive circuler alternativement entre l'une et l'autre. L'intention n'était pas implicite au moment de la conception, mais s'est imposée d'elle-même, du fait des contraintes de disponibilité des espaces à l'écomusée (une salle et un couloir) car, au bout du compte et aux yeux de l'institution, il est devenu un seul et même projet, pensé sur plusieurs années et construit, comme une vie, en strates successives.

De la collaboration avec les artistes résulte un parcours d'exposition mais aussi des rencontres intergénérationnelles dans et autour de l'exposition, des publics nouveaux pour l'écomusée, lémergence de réflexions autour de la notion d'estime de soi, etc. Dans ce projet polyphonique, les uns ont accepté directement, sans fard, de confier leurs histoires de vies à des artistes qui ont, c'est un des pouvoirs de l'art, transposé et sans doute transcendé ces témoignages pour donner à voir ou à entendre l'indicible et l'invisible.

Au demeurant, quels qu'aient été les motifs de leurs départs respectifs - politiques, économiques, culturels ou sentimentaux -, que la France ait été une destination choisie ou subie, ces parcours de vie illustrent les luttes, les espoirs, les quêtes et les revendications des migrants d'hier et d'aujourd'hui. Ils bousculent parce qu'ils questionnent nos pratiques; ils disent, aussi, la force et l'attractivité d'une démocratie stable.

Le livre d'or de l'écomusée, les réactions des visiteurs et les échanges avec les publics accueillis dans le cadre d'actions de médiation élaborées avec les scolaires, les associations d'insertion, les services des communes qui mobilisent des personnels pour développer des programmes à caractère social démontrent que ce projet ne laisse pas indifférent, peut-être parce que, comme l'a souligné chacun des partenaires mobilisés par l'écomusée, ces histoires résonnent avec celle de chacun d'entre nous. En ce sens, c'est certain, le territoire Creusot Montceau est monde ${ }^{8}$. 